\title{
Effect and Mechanism of Carbamazepine on SCN1A Expression in Temporal Lobe Epilepsy Rats
}

LIU ZHANG, YONG JIAN FENG, WEI LI, YONG JUN YI, JING WANG AND XIANG YU WANG*

Department of Neurosurgery, The First Affiliated Hospital of Jinan University, Guangdong 510630, China

Zhang et al.: Effect of Carbamazepine on SCN1A Expression in Epilepsy Rats

To investigate the effect of carbamazepine treatment on the expression of SCN1A in temporal lobe epilepsy and its mechanism of action. 90 male Wistar rats were randomly divided into 30 rats each in the carbamazepine group, model group and blank control group, in which the temporal lobe epilepsy rat model was established using lithium-pilocarpine in the carbamazepine and model groups. After successful modeling, the carbamazepine group was given carbamazepine solution at a concentration of $16 \mathrm{mg} / \mathrm{ml}$ in a dose of $2 \mathrm{ml}$ once/d; the model group was not given any drug treatment, and distilled water was given $2 \mathrm{ml} /$ time/d. The normal control group was fed routinely without any special treatment. Immunohistochemistry, quantitative reverse transcription polymerase chain reaction and Western blot assay were used to detect the changes of SCN1A expression level in each group. Polymerase chain reaction results showed that the expression of SCN1A messenger ribonucleic acid in the model group was significantly higher than that in the control group and the carbamazepine group, and the difference was statistically significant $(p<0.05)$, and the SCN1A messenger ribonucleic acid in the carbamazepine group was higher than that in the control group, but the difference was not statistically significant $(p>0.05)$. The level of SCN1A protein product Nav1.1 in the model group was significantly higher than that in the control group and the carbamazepine group, and the difference was statistically significant, although the level of SCN1A protein product Nav1.1 in the carbamazepine group was higher than that in the control group, but the difference was not statistically significant, indicating that the administration of carbamazepine could reduce the expression of CN1A protein product Nav1.1 level. Immuno-histochemical results showed that SCN1A was more expressed in the temporal cortex and CA1 and CA3 regions of the hippocampus in normal rats. Compared with the control group, the expression of SCN1A immuno positive cells in the hippocampal CA1 and $\mathrm{CA} 3$ regions and temporal cortex of the model group was increased, with highly significant difference $(p<0.01)$; no significant difference $(p>0.05)$ was found in the carbamazepine group. Carbamazepine could better control the abnormally elevated expression of SCN1A in temporal lobe epileptic rats and return its expression level to normal, which may be related to the restoration of the impaired inward rectification by reducing the expression of Nav1.1 channel protein and thus buffering the extracellular sodium load to some extent. Maintaining resting membrane potential reduces neuronal excitability ultimately may reduce the generation of epileptiform discharges.

Key words: Carbamazepine, SCN1A, temporal lobe epilepsy, rat model

Epilepsy, a chronic encephalopathy caused by a variety of causes, causes excessive and repeated super synchronization discharge of brain neurons, which leads to transient brain dysfunction. Epidemiological investigation shows that the prevalence rate is $5 \%$, and there are about 6-7 million patients in China ${ }^{[1]}$. Temporal lobe epilepsy (TLE) is one of the common intractable epilepsy, accounting for more than $50 \%$ of the intractable epilepsy. Therefore, it is of great significance to study new and effective ways and methods for the treatment of intractable epilepsy.

Clinical studies have found that carbamazepine has definite curative effect on epilepsy, and has multi- target and multi-channel comprehensive regulatory effect $^{[2,3]}$. As a commonly used drug in the clinical treatment of temporal lobe epilepsy, carbamazepine has a good clinical effect in the treatment of temporal lobe epilepsy $^{[4]}$. Voltage gated sodium channels mainly play the role of maintaining cell resting membrane potential and regulating cell excitability, which may play an important role in the pathogenesis of epilepsy ${ }^{[5-7]}$. There are many members of voltage-gated natriuretic channel subfamily. At present, in the brain tissues of patients with intractable glume epilepsy, it is found that the decreased expression level of SCN1A gene expression product Nav1.1 channel and the decreased

*Address for correspondence

E-mail: zhbxjisn788@163.com 
inward rectifying sodium current are closely related to the occurrence of epilepsy ${ }^{[8-10]}$. In this study, the expression of SCN1A in hippocampus of pilocarpine induced temporal lobe epilepsy rat model was detected to explore the therapeutic effect and mechanism of carbamazepine on temporal lobe epilepsy, and to provide more scientific and accurate theoretical basis for clinical treatment.

\section{MATERIALS AND METHODS}

\section{Experimental animal:}

A total of 90 healthy male Wistar rats, aged from 6 to $8 \mathrm{w}$, weighing $230 \pm 10 \mathrm{~g}$, were randomly divided into carbamazepine group, model group and blank control group, with 30 rats in each group.

\section{Construction of chronic epilepsy model:}

Rats were reared in a single cage for one $\mathrm{w}$ to adapt to the environment. The behavior of rats was observed daily, and the rats with spontaneous seizures were excluded. After randomly divided into three groups, the temporal lobe epilepsy model was established with lithium pilocarpine. In the model group (and carbamazepine group), $1.27 \%$ lithium chloride $127 \mathrm{mg} / \mathrm{kg}(12.7 \mathrm{mg} / \mathrm{ml})$ was injected intraperitoneally on the first day, then atropine hydrochloride injection 1 $\mathrm{mg} / \mathrm{kg}$ was injected intraperitoneally $18-20 \mathrm{~h}$ later, $1 / 2$ of $1 \%$ pilocarpine $150-200 \mathrm{mg} / \mathrm{kg}$ diluted by normal saline was injected intraperitoneally $30 \mathrm{~min}$ later, and $1 / 2$ of $1 \%$ pilocarpine $150-200 \mathrm{mg} / \mathrm{kg}$ diluted by normal saline was injected intraperitoneally $10 \mathrm{~min}$ later. The convulsion performance of SD rats was determined according to Racine standard.

\section{Modeling success criteria:}

Seizures of Racine-IV or above were considered as successful models. If grade III or below reaction occurred $30 \mathrm{~min}$ after the second administration, $1 / 3$ of the total dose was given to continue to induce epileptic seizures until epilepsy was induced (raceIV or above). After about one $\mathrm{h}$ of status epilepticus, diazepam injection $10 \mathrm{mg} / \mathrm{kg}$ was given immediately to terminate the seizure. When diazepam was injected intraperitoneally, $300 \mathrm{mg} / \mathrm{kg}$ levetiracetam diluted with normal saline was injected intraperitoneally. If the control was not ideal, $10 \mathrm{mg} / \mathrm{kg}$ diazepam injection could be injected 20 min later.

\section{Administration:}

Carbamazepine group: the concentration of carbamazepine solution was $16 \mathrm{mg} / \mathrm{ml}$, the dose was
$2 \mathrm{ml}$, once a day. Model group: after the establishment of the model, no drug treatment was given, and distilled water $2 \mathrm{ml} /$ time/d was given. Control group: conventional feeding, no special treatment. After $8 \mathrm{w}$ (60 d) of chronic phase, the rats in each group were treated by gavage for $2 \mathrm{w}(14 \mathrm{~d})$.

\section{Sampling and slice preparation:}

After the successful establishment of temporal lobe epilepsy model, the brain tissues were collected and sectioned at $0 \mathrm{~h}, 6 \mathrm{~h}, 72 \mathrm{~h}$ and $2 \mathrm{w}$ after the termination of epilepsy. The specific production process is as follows: after anesthesia, SD rats were decapitated quickly and the brain was separated on ice. Half of the hippocampal tissue was about $10 \mathrm{mg}$, each of which was added to the EP tube and stored at $-80^{\circ}$. The other half was perfused with heparin and normal saline, then perfused with 4 $\%$ paraformaldehyde, 40 drops per min, fixed for 30 min until the liver became hard. The whole brain tissue was cut and stored in $4^{\circ}-4 \%$ paraformaldehyde for $7 \mathrm{~d}$. The fixed brain was sliced by vt1200s, a Leica automatic vibration slicer, with a thickness of $30 \mu \mathrm{m}$, and stored in $4^{\circ}$ - glycol antifreeze anti-freeze.

\section{Immunohistochemical staining:}

The paraffin sections were soaked in Phosphate Buffered Saline (PBS) solution for 3 times. The slices were sealed with sheep serum for $60 \mathrm{~min}$ at room temperature. Then, Rabbit anti rat SCN1A polyclonal antibody (1:50 dilution) was added and put into $4^{\circ}$ refrigerator overnight. Then PBS solution was soaked for 3 times. Goat anti mouse antibody labeled with horseradish peroxidase (HRP) was added and incubated at $37^{\circ}$ for $30 \mathrm{~min}$. PBS solution, 3 times. $1 \mathrm{~mol} / 1$ Tris $\mathrm{HCl}$ buffer for $5 \mathrm{~min}$. $0.05 \%$ 3,3'-diaminobenzidinetetrahydrochlorid $+0.05 \mathrm{~mol} / \mathrm{l}$ Tris $\mathrm{HCl}$ buffer was added to the slices, and 1-2 drops of $3 \%$ hydrogen peroxide was added to develop the color. The reaction took 5-15 min, and the color reaction was observed and monitored under the microscope. $0.05 \mathrm{~mol} / 1$ Tris $\mathrm{HCl}$ buffer was used to terminate the reaction and wash out the non-specific staining. Wash with distilled water. Re dyeing with hematoxylin for 2 3 min, washing for 10 min. Dehydrated with alcohol, transparent with xylene, sealed with neutral gum, observed under microscope.

\section{Western blot analysis:}

A total of $3 \mathrm{mg}$ fresh brain tissue was added into 100 UL Ripa lysate, and the supernatant was removed after centrifugation. Bicinchoninic acid concentration was 
used to quantitatively detect protein concentration, and then $30 \mu \mathrm{g}$ protein/well was loaded. After SDS-PAGE electrophoresis, it was transferred to Polyvinylidene fluoride membrane, sealed with $5 \%$ Bovine serum albumin at room temperature for $2 \mathrm{~h}$, incubated with SCN1A working solution at $4^{\circ}$ overnight, washed with tris-buffered saline and Tween 20 (TBST) at room temperature for 3 times, and incubated with HRP labeled working solution for $2 \mathrm{~h}$. After 3 times of cleaning, the fresh Enhanced chemiluminescence (ECL) solution was added. The high performance luminescent gel imaging system was exposed. The gray scale of the band was calculated by image $J$ software, and the relative expression intensity of phosphorylated protein was compared with the ratio of phosphorylated protein to total protein.

\section{Real time quantitative PCR:}

Total RNA was isolated using Trizol reagent according to the manufacturer's instructions. Reverse transcription of RNA was performed with primescript TM RT kit. SYBR Green (rr420a, Takara) was used for quantitative real-time PCR. The multiple change is calculated using the formula $R Q=2^{-\Delta \Delta C t}$. The sequence of primers was as follows: SCN1A F: 5'-TCATGCTGAAGCTGTTCGA CT-3', SCN1A R: 5'-CTTCTGTTCTTCTGTCATAA-3'; $\beta$-actin F: 5'-CACTGGCATCGTGATGGA-3' and $\beta$-actin R: 5'-GGCCATCTCTTGCTCGAA-3'.

\section{Statistical analysis:}

All the experimental data were analyzed by SPSS 22.0 software. The data were expressed as $\mathrm{x} \pm \mathrm{s}$. The data between groups were compared by $\chi^{2}$ analysis of variance, and the data between groups were compared by t test. $* \mathrm{p}<0.05, * * \mathrm{p}<0.01$ were considered statistically significant.

\section{RESULTS AND DISCUSSION}

During the experiment, a total of 90 rats were intraperitoneally injected with lithium chloride pilocarpine hydrochloride chemical kindling method to prepare chronic epilepsy model. No rats died in the acute phase $(24 \mathrm{~h})$. During the incubation period $(2 \mathrm{w}), 11$ rats died of chronic failure due to frequent convulsions, infection, ascites and inability to eat autonomously. Most of them died within $1 \mathrm{w}$ after epilepsy, with a mortality rate of $18.33 \% .49$ surviving epilepsy induced epileptic rats entered the chronic phase, and no death occurred in the chronic phase and administration phase. In the normal group, 30 rats were intraperitoneally injected with normal saline as the control, and all survived during the experiment. A total of 79 rats survived and reached the end of the experiment, including 24 rats in the carbamazepine group, 25 rats in the model group and 30 rats in the blank control group. Compared with the model group, the total attack times and attack time of carbamazepine group were significantly reduced $(\mathrm{p}<0.05)$, as shown in Table 1.

We compared and analyzed the relative expression of SCN1A mRNA in hippocampus of rats in control group, model group and carbamazepine group. The results showed that the expression of SCN1A mRNA in the model group was significantly higher than that in the control group and carbamazepine group $(\mathrm{p}<0.05)$, and the SCN1A mRNA in the carbamazepine group was higher than that in the control group, but there was no significant difference between the two groups $(\mathrm{P}>0.05)$, indicating that the expression of SCN1A decreased after carbamazepine administration, as shown in fig. 1.

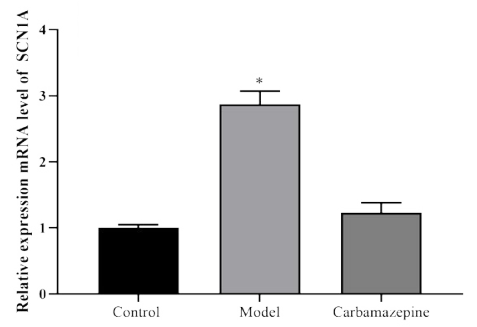

Fig. 1: Expression of SCN1A mRNA in hippocampus of temporal lobe epilepsy rats

Note: Compared with the control and carbamazepine group, $* \mathbf{p}<\mathbf{0 . 0 5}$.

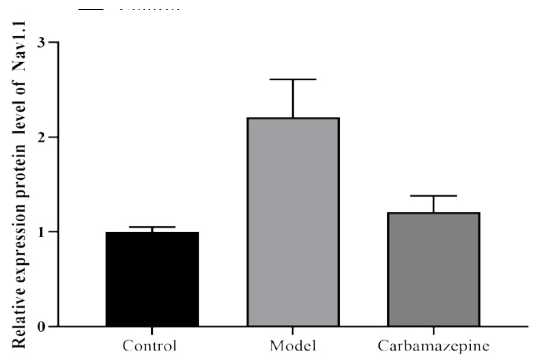

Fig. 2: Levels of SCN1A protein product Nav1.1 in hippocampus of temporal lobe epilepsy rats in each group

Note: Compared with the control group, *p<0.05.

TABLE 1: PREPARATION AND SURVIVAL OF EPILEPTIC RAT MODEL

\begin{tabular}{lccc}
\hline Group & N & Seizure frequency (Times) & Total seizure duration (min) \\
\hline Model & 25 & $349.60 \pm 79.30$ & $1789.84 \pm 257.38$ \\
Carbamazepine & 24 & $237.10 \pm 54.40^{*}$ & $1291.40 \pm 261.80^{*}$ \\
\hline
\end{tabular}

Note: Compared with the model group, ${ }^{*} \mathrm{p}<0.05$. 
Western blot results showed that the level of Nav1.1 protein product of SCN1A in hippocampus of model group was significantly higher than that of control group and carbamazepine group. In addition, although the level of SCN1A protein product Nav1.1 in the carbamazepine group was higher than that in the control group, the difference was not statistically significant, indicating that carbamazepine can reduce the expression of CN1A protein product Nav1.1, as shown in fig. 2.

The results of immunohistochemistry showed that SCN1A was highly expressed in the cortex of temporal lobe and CA1, CA3 of hippocampus in normal rats. Compared with the control group, the expression of SCN1A immunoreactive cells in hippocampal CA1 area, CA3 area and temporal cortex of the model group were significantly increased $(\mathrm{P}<0.01)$; there was no significant difference between the carbamazepine group and the control group ( $\mathrm{P}>0.05)$, as shown in fig. 3 .

Ion channels are a large class of membrane binding proteins, which are essential for maintaining the normal function of nucleated cells, because they determine the excitability of the membrane. Recent studies have found that the dysfunction of ion channels in the nervous system will have a serious impact on the body ${ }^{[11,12]}$. Recent studies have found that ion channel abnormalities related to epilepsy are mainly concentrated in potassium, sodium and calcium ions, and epilepsy genes affecting ion channels almost all cause seizures through the abnormal transmembrane movement of these ions ${ }^{[13]}$. Numerous studies have proved that the occurrence of epilepsy is related to the abnormal function of sodium channel, and the pathogenesis of epilepsy is related to the congenital mutation of sodium channel gene and acquired sodium channel disease ${ }^{[14-16]}$. Several types of idiopathic epilepsy have been confirmed to be caused by mutations in the VGSCs gene. Mutated sodium channel gene can change the function of sodium channel, such as promoting sodium channel activation, accelerating its reactivation, slowing its inactivation, etc. Acquired sodium channel abnormalities mainly cause epilepsy by changing the expression and function of sodium

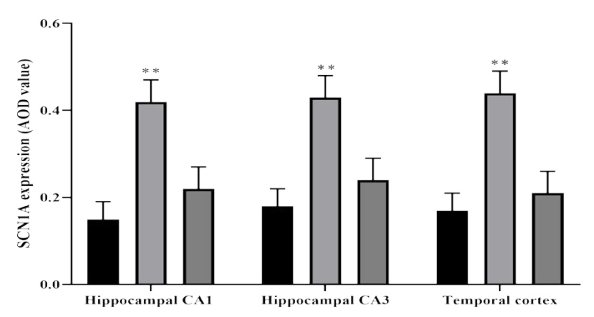

Fig. 3: Expression of SCN1A in hippocampus and temporal lobe of rats

Note: Compared with the control group, ${ }^{* *} \mathbf{p}<\mathbf{0 . 0 1}$. channels, such as increasing the sustained sodium current (INAP), which increases the excitability of neurons ${ }^{[15,17]}$. Animal model studies in mice have found that SCN1A gene mutation can produce ataxia, dystonia, tremor, progressive paralysis and other movement disorders ${ }^{[18-20]}$, but there is no epileptic seizure. The decrease of the number of Nav1.1 in heterozygous individuals can reduce the excitability of neurons ${ }^{[21]}$. Using PCR technique, we found that compared with the control group, the expression level of SCN1A in the hippocampus of temporal lobe epilepsy rats was abnormally increased, and the protein product Nav1.1 level of SCN1A was also significantly increased, and the positive rate of SCN1A in the hippocampus neurons was significantly increased. These results indicate that the abnormal expression of SCN1A gene is related to the pathogenesis of temporal lobe epilepsy in rats. It is found that the enhanced dependence of sodium channel Nav1.1 and the generation of characteristic "reactivated" sodium current are also suitable for maintaining the stable conduction of high-frequency action potential, which is conducive to the propagation of epileptiform discharge during seizures. Therefore, we suggest that the increase in the number of the acquired sodium channels may increase the excitability of neurons and thus participate in the generation and transmission of epileptiform discharges.

It has become a consensus that antiepileptic drugs play a role by acting on ion channels. At present, a variety of antiepileptic drugs with this effect have been used in clinical practice. For example, tinidapt mainly acts on potassium channels ${ }^{[22]}$, valproic acid and lamotrezol have calcium channel inhibition ${ }^{[23]}$. However, sodium channel opener is still in the primary stage of experimental research. As the first discovered sodium channel opener, phenytoin can inhibit epileptic discharges and reduce seizures caused by neurotoxins. However, sodium channels also exist widely in the heart and blood vessels. Studies have shown that phenytoin has significant peripheral side effects, and its clinical application is limited. Carbamazepine is a traditional antiepileptic drug, which can act on nerve cell membrane, reduce the permeability of nerve cell membrane to $\mathrm{Na}^{+}$and $\mathrm{Ca}^{2+}$ inhibit nerve cells, reduce their excitability, and control seizures. It has been found that carbamazepine is a specific opener of Nav1.1 channel. Meanwhile, it is also the first discovery of exogenous opener for Nav1.1 channe ${ }^{[24,25]}$. It can significantly increase Nav1.1 channel current, hyperpolarize cell membrane and increase the stability of cell membrane. Therefore, it is speculated that 
carbamazepine may have therapeutic effect on the pathophysiological process involving Nav1.1 channel. Up to now, the relationship between carbamazepine and Nav1.1 channel and epilepsy has not been reported. In this study, we established a rat model of temporal lobe epilepsy, and detected the expression of SCN1A mRNA, protein level and immune positive rate by PCR, WB and immunohistochemistry. The results showed that after the administration of carbamazepine, the expression of SCN1A mRNA, protein level and immune positive rate in the carbamazepine group were significantly lower than those in the model group, and the difference was statistically significant, which indicated that carbamazepine could effectively inhibit the expression of SCN1A. In addition, compared with the control group, although the expression level was higher than that of the control group, the difference was not statistically significant, indicating that carbamazepine has a good effect on epilepsy caused by SCN1A.

In conclusion, carbamazepine can significantly control the abnormal increase of SCN1A in temporal lobe epilepsy rats, and make its expression level return to normal level. This may be due to the reduction of Nav1.1 channel protein expression, which can restore the damaged inward rectification to a certain extent, so as to buffer the extracellular sodium load, maintain the resting membrane potential, reduce the excitability of neurons, and ultimately reduce the production of epileptic discharges.

\section{Acknowledgements:}

This work was supported by The First Affiliated Hospital of Jinan University.

\section{Conflicts of interest:}

The authors report no conflicts of interest.

\section{REFERENCES}

1. Reyes A. Influence of dendritic conductances on the input-output properties of neurons. Annu Rev Neurosci 2001;24(1):653-75.

2. Cossart R, Dinocourt C, Hirsch JC, Merchan-Perez A, De Felipe J, Ben-Ari Y, et al. Dendritic but not somatic GABAergic inhibition is decreased in experimental epilepsy. Nat Neurosci 2001;4(1):52-62.

3. Bernard C, Anderson A, Becker A, Poolos NP, Beck H, Johnston D. Acquired dendritic channelopathy in temporal lobe epilepsy. Science 2004;305(5683):532-5.

4. Graves TD, Hanna MG. Neurological channelopathies. Postgrad Med J 2005;81(951):20-32.

5. Winawer MR. Epilepsy Genetics. Neurologist 2002;8(3):133-51.

6. Felix R. Channelopathies: ion channel defects linked to heritable clinical disorders. J Med Genet 2000;37(10):729-40.
7. Chyung AS, Ptácek LJ. Genetics of epilepsy. CONTINUUM: Lifelong Learning in Neurology 2005;11(2):79-94.

8. Armijo JA, Shushtarian M, Valdizan EM, Cuadrado A, Adin J. Ion channels and epilepsy. Curr Pharma Des 2005;11(15):19752003.

9. Yamakawa K. Epilepsy and sodium channel gene mutations: gain or loss of function?. Neuroreport 2005;16(1):1-3.

10. Stafstrom CE. Persistent sodium current and its role in epilepsy. Epilepsy Curr 2007;7(1):15-22.

11. Meisler MH, Kearney JA. Sodium channel mutations in epilepsy and other Neurological disorders. J Clin Invest 2005;115(8): 2010-17.

12. Sprunger LK, Escayg A, Tallaksen-Greene S, Albin RL, Meisler MH. Dystonia associated with mutation of the neuronal sodium channel Scn8a and identification of the modifier locus Scnm1 on mouse chromosome 3. Hum Mol Genet 1999;8(3):471-9.

13. Smith MR, Goldin AL. A mutation that causes ataxia shifts the voltage-dependence of the Scn8a sodium channel. Neuroreport 1999;10(14):3027-31.

14. Yogeeswari P, Ragavendran JV, Thirumurugan R, Saxena A, Sriram D. Ion channels as important targets for antiepileptic drug design. Curr Drug Targets 2004;5(7):589-602.

15. Graves TD, Hanna MG. Neurological channelopathies. Postgrad Med J 2005;81(951):20-32.

16. Goldin AL. Evolution of voltage-gated $\mathrm{Na}^{+}$channels. J Exp Biol 2002;205(5):575-84.

17. Afshari FS, Ptak K, Khaliq ZM, Grieco TM, Slater NT, McCrimmon DR, et al. Resurgent Na currents in four classes of neurons of the cerebellum. J Neurophysiol 2004;92(5):2831-43.

18. Do MT, Bean BP. Sodium currents in subthalamic nucleus neurons from Nav1. 6-null mice. J Neurophysiol 2004;92(2):726-33.

19. Khaliq ZM, Gouwens NW, Raman IM. The contribution of resurgent sodium current to high-frequency firing in Purkinje neurons: an experimental and modeling study. J Neurosci 2003;23(12):4899-912.

20. Audra VW, Gary M. Impaired Firing and Cell-Specific Compensation in Neurons Lacking Nav1.6 Sodium Channels. J Neurosci 2006;26(27):7172-80.

21. Astman N, Gutnick MJ, Fleidervish IA. Persistent sodium current in layer 5 neocortical neurons is primarily generated in the proximal axon. J Neurosci 2006;26(13):3465-73.

22. Zhou W, Goldin AL. Use-dependent potentiation of the Nav1. 6 sodium channel. Biophys J 2004;87(6):3862-72.

23. Trudeau MM, Dalton JC, Day JW, Ranum LP, Meisler MH. Heterozygosity for a protein truncation mutation of sodium channel SCN8A in a patient with cerebellar atrophy, ataxia, and mental retardation. J Med Genet 2006;43(6):527-30.

24. Reckziegel G, Beck H, Schramm J, Urban BW, Elger CE. Carbamazepine effects on $\mathrm{Na}+$ currents in human dentate granule cells from epileptogenic tissue. Epilepsia 1999;40(4):401-7.

25. Kuo CC, Chen RS, Lu LU, Chen RC. Carbamazepine inhibition of neuronal $\mathrm{Na}+$ currents: quantitative distinction from phenytoin and possible therapeutic implications. Mol Pharmacol 1997;51(6):1077-83.

This is an open access article distributed under the terms of the Creative Commons Attribution-NonCommercial-ShareAlike 3.0 License, which allows others to remix, tweak, and build upon the work non-commercially, as long as the author is credited and the new creations are licensed under the identical terms

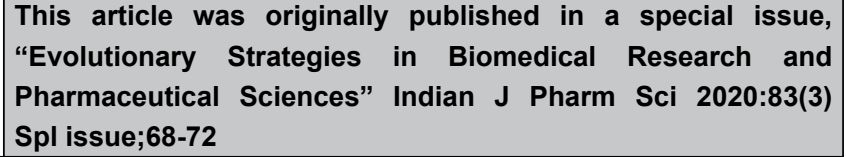

